\title{
Scaling Laws for MEMS Mirror-Rotation Optical Cross Connect Switches
}

\author{
Richard R. A. Syms, Member, IEEE
}

\begin{abstract}
The design of large-scale mirror-rotation free-space optical cross-connect switches based on arrays of microelectromechanical torsion mirrors is considered. The layout of a compact switch is first presented. The parameters of the Gaussian beam that maximizes the port count for a given mirror turn angle is then identified, and the supporting optical system needed to create the desired beam is defined. Scaling laws for the optical path length needed for a given number of ports are then derived. Numerical simulations are used to verify the ideal configuration, and scaling laws are proposed for various departures from the ideal. It is shown that ideal operation can still be maintained when the mirrors are curved, and operating conditions that minimize the effect of mirror curvature are identified.
\end{abstract}

Index Terms-MEMS, MOEMS, optical switching.

\section{INTRODUCTION}

$\mathbf{T}$ RANSPARENT space-division optical switches are needed for rerouting signals in broad-band fiber communications systems. The requirements for such switches include low insertion loss, low crosstalk, insensitivity to wavelength and polarization, high power handing capability and scalability to large port counts. Based on these criteria, it has been demonstrated that switches based on the deflection of free-space beams by small moving mirrors can outperform switches based on interferometric combination of guided waves.

Free-space optical switches generally use silicon or polysilicon mirrors fabricated using microelectromechanical systems (MEMS) technology. Actuation can be by electrothermal, electrostatic, magnetic, electromagnetic, or piezoelectric forces. Individual MEMS components have been demonstrated as $1 \times 1$ reflective switches [1]-[4], $2 \times 2$ reflective switches [5], and $2 \times 2$ transmissive switches [6]-[10]. In each case, the switch is operated by the insertion or removal of a small mirror.

Switch arrays are constructed from multiple switch elements. The arrangement usually follows one of three configurations: two-dimensional (2-D) matrices of $N \times N$ two-position mirrors, linear arrays of $N \times N$ single-axis multiple-position mirrors [three-dimensional (3-D) $1 \times N$ arrays], and 2-D arrays of $N^{2} \times$ $N^{2}$ dual-axis multiple-position mirrors (3-D $2 N$ arrays).

Fig. 1 shows the arrangement of the first type of cross connect. The inputs are provided by a linear array of $N$ optical fibers. Light emerging from the fiber array is collimated by a linear array of lenses into a set of quasi-parallel beams that propagate in free space. The outputs are taken from a similar array

Manuscript received January 15, 2002; revised March 18, 2002. This work was supported by the Micromachined Product Division of Analog Devices Inc.

The author is with the Optical and Semiconductor Devices Section, Department of Electrical and Electronic Engineering, Imperial College, London SW7 2BT, U.K. (e-mail: r.syms@ic.ac.uk).

Publisher Item Identifier 10.1109/JLT.2002.800339.

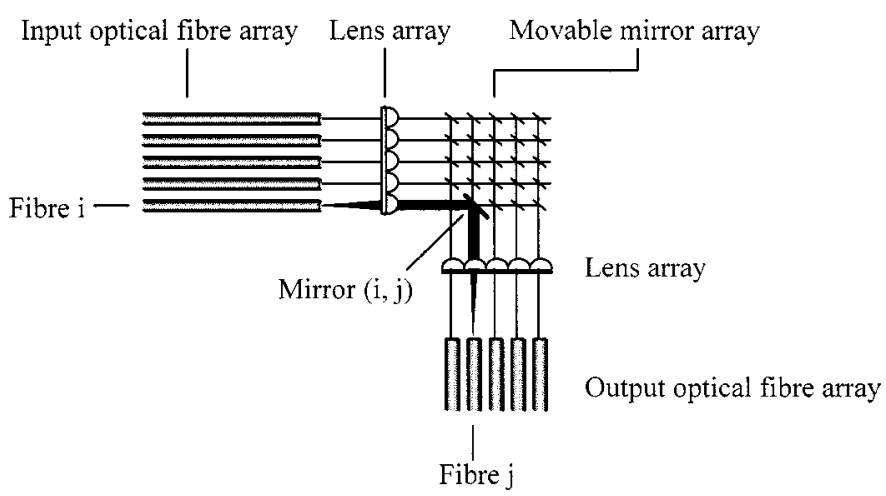

Fig. 1. Principle of $N \times N$ mirror-insertion free-space optical cross connect.

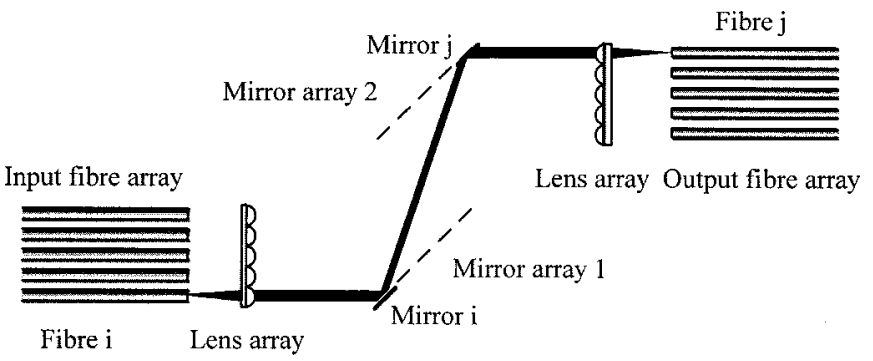

Fig. 2. Principle of an $N \times N$ mirror-rotation free-space optical cross connect.

of fibers, equipped with a similar set of lenses, and designed to accept a similar set of beams. The axes of the input and output fibers are typically arranged at right angles.

The space between the input and output fiber arrays is filled with a set of small movable mirrors, capable of being inserted and removed from the intersections between the beams at an angle intermediate between their directions. A path between input fiber $i$ and output fiber $j$ is then established by the insertion of the relevant mirror $M(i, j)$.

$N \times N$ free-space mirror insertion optical cross-connect switches have been constructed using mirrors that are translated into position on elastic linear suspensions [11], rotated into position on elastic torsion suspensions [12], [13], rotated into position on freely pivoting hinged mounts [14]-[18], or elevated out-of-plane [19].

Fig. 2 shows the second type of cross connect. The inputs and outputs are again linear arrays of $N$ fibers equipped with collimators. However, between the input and output, the beams are reflected from two linear arrays of mirrors. Each individual mirror may be rotated through a variable angle about an axis normal to the figure. A path between input fiber $i$ and output fiber $j$ is then established by angular adjustment of mirror $i$ from 


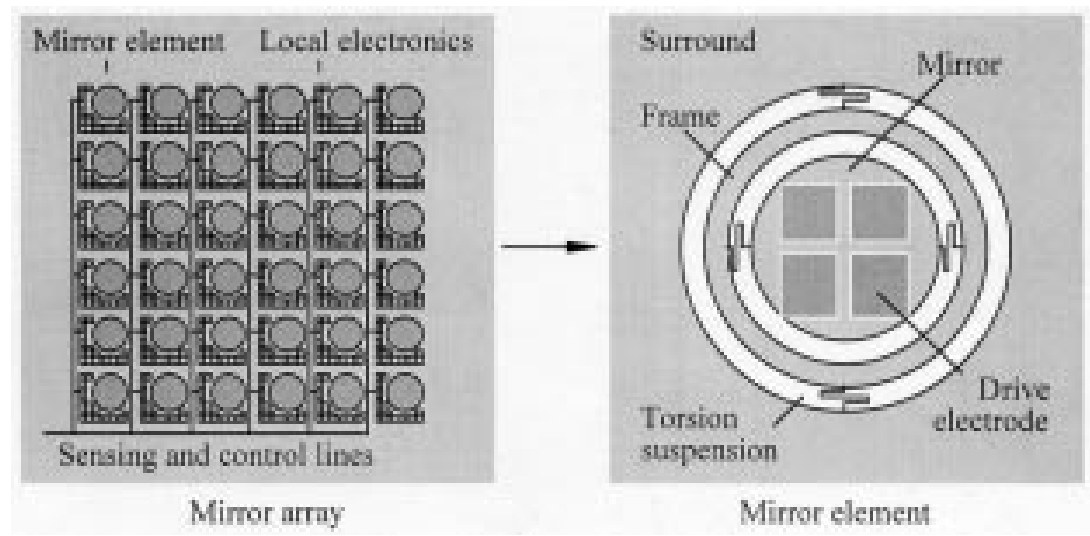

Fig. 3. Moving mirror switch based on a two-axis gimbal-mounted torsion mirror.

the first array and mirror $j$ from the second, in a periscope configuration. $N \times N$ cross connects have been constructed using MEMS mirrors on torsion suspensions [20]-[22].

A similar principle is used in the third type of cross connect. The linear arrays of $N$ fibers and collimators each replaced with 2-D arrays of $N^{2}$ fibers and collimators, and the linear arrays of $N$ single-axis mirrors are replaced by 2-D arrays of $N^{2}$ dual-axis mirrors. The required mirror motion is achieved by mounting the mirror on a gimbal suspension, as in Fig. 3. This type of switch is scalable to a higher port count, and has been demonstrated using several forms of MEMS mirror [23]-[28].

The number of ports supported by a mirror-rotation cross connect is limited by the diameter of the beams and the angle through which the mirrors may turn. For electrostatically driven mirrors on elastic suspensions, the turn angle is restricted by a nonlinear snap-down phenomenon [29]. This problem may be alleviated by a nonlinear elastic suspension [30]. Alternatively, a comb drive may be used instead of a parallel plate drive [31]-[33].

In principle, the number of ports obtained for a given turn angle can be raised by increasing the separation between the mirror planes, because a mirror on one plane will then subtend a smaller angle at the other. However, this strategy also involves an increase in the optical path. Because the beams are narrow, they diverge as they propagate according to the laws of diffraction, so increasing the path will increase the beam diameter. Larger mirrors are then required for efficient reflection, which nullifies the effect of placing the mirror planes farther apart. Maximization of the port count therefore involves identification of the most effective optical layout rather than an increase in the overall size of the switch.

For mirror insertion switches (as in Fig. 1), an improved layout is obtained by using staggered fiber arrays or staggered refractive components to equalize the optical paths [34], [35]. The effect of divergence can also be reduced, by constructing a large array from several smaller ones that are linked together by lenses that recollimate the beams [36]. The parameters of individual components such as lenses and mirrors can also be optimized by using numerical simulations of beam propagation, and this has been done for mirror insertion [18] and mirror-rotation switches [22].

Any effective optical layout must provide good performance under different operating conditions. For example, MEMS mirrors are often constructed as multilayers, using a metal (e.g., gold) to improve the reflectivity of a silicon substructure. Any stress in the deposited film will cause the mirror surface to become curved. The beam reflected from such a mirror may then diverge, so that it is no longer reflected efficiently by the next mirror.

Even if static curvature is eliminated, dynamic curvature may arise in operation. All metals are imperfect reflectors, and absorb a fraction of the incident power. At near-infrared wavelengths, the fraction is $2 \%-3 \%$ for Au. The absorbed power will heat the mirror until a balance is reached between the rate of optical heating and the rate of cooling through the suspension and the surrounding gas [37], [38]. Since the expansion coefficients of the metal and its support differ, the mirror will act as a thermal bimorph and become curved. Environmental heating will have a similar effect. Although double-sided metallization is a solution, it is useful to identify designs that are immune to mirror curvature.

In this paper, we consider the design of mirror-rotation cross connects, using analytic methods that are complementary to earlier work [18], [22]. In Section II, we focus on a simple layout consisting of opposing planes of tilt mirrors. We use a simplified unfolded model of the system, which ignores off-axis effects such as astigmatism, and neglect mirror-pointing errors. Using analytic formulas for the propagation of Gaussian beams, the parameters of the beam that maximizes the port count for a given mirror turn angle is first identified. To our knowledge, the significance of this optimized beam has not been highlighted before. The supporting optics needed to create the desired beam are defined, and scaling laws for the optical path length needed for a given number of ports are derived.

In Section III, a simple numerical algorithm for beam propagation is defined. In Section IV, this algorithm is used to verify the earlier design rules, and further scaling laws are established for system imperfections. Finally, operating configurations that minimize the effect of mirror curvature are identified. It is shown that high throughput may be established even with imperfect mirrors, and a simple analytic proof of the required conditions is given in Section V. Because $N \times N$ and $N^{2} \times N^{2}$ cross connects (3-D $1 \times N$ and 3-D $2 N$ arrays) have similar layouts, the designs are relevant to both types of switch.

\section{THEORETICAL ANALYSIS}

We begin by defining a simple layout for a mirror-rotation optical cross-connect switch. Fig. 4 shows a number of possibilities. In each case, the mirror planes are assumed to consist 

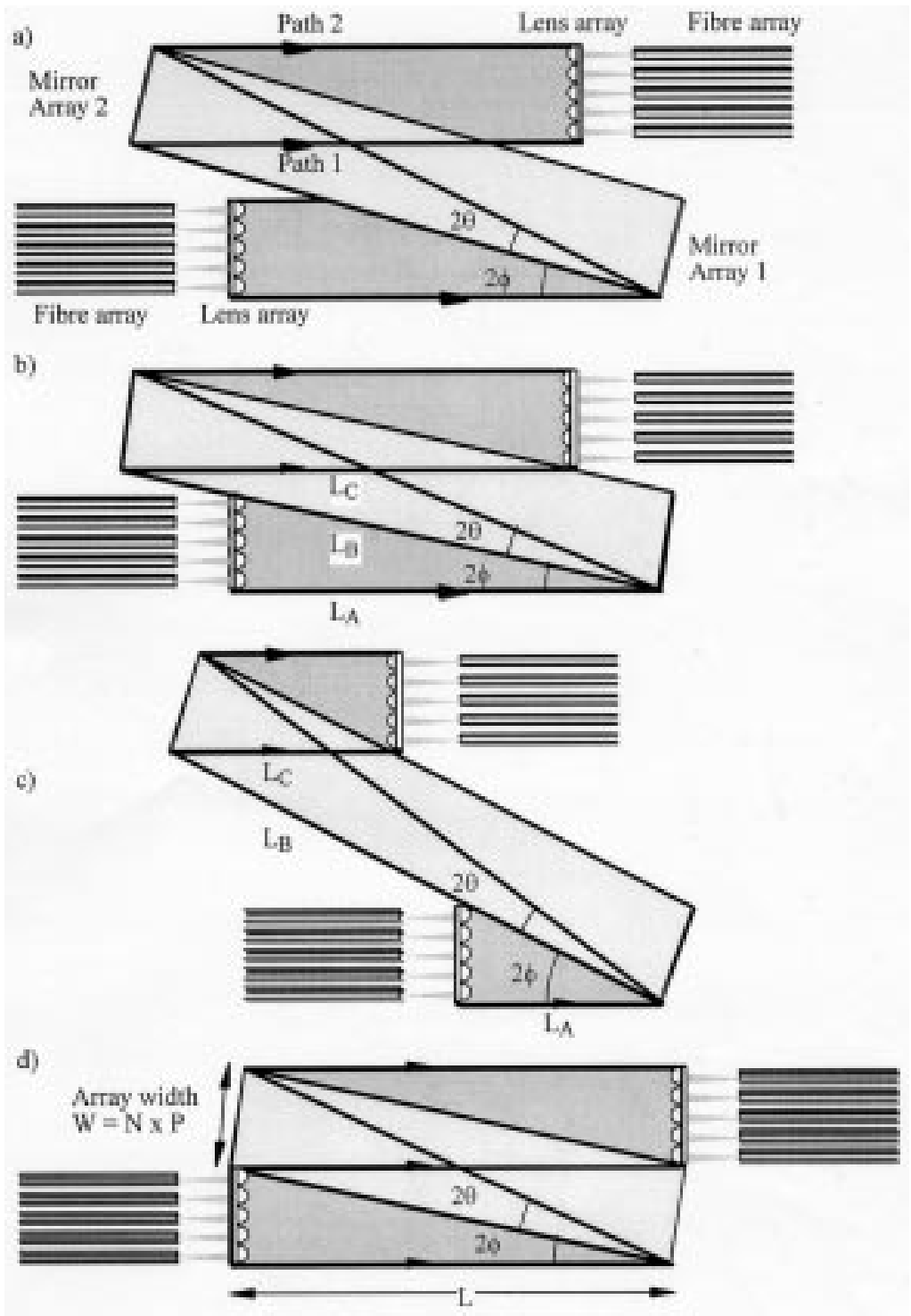

Fig. 4. Various layouts for a mirror-rotation free-space optical cross connect.

of either a linear array of $N$ single-axis mirrors or a 2-D array of $N^{2}$ mirrors, as appropriate. The range and sense of turn angle of each mirror varies with its position in the array. The central mirror must turn through both positive and negative angles. The outermost mirrors must turn through approximately twice the maximum angle of the central mirror, but only in one sense. Each mirror has a maximum turn angle of $\pm \theta$ about either axis. For comb drives, these maximum angles are decoupled; for parallel-plate drives, they are not. Here, we assume the former case.

In Fig. 4(a), the restricted turn angle implies (to reasonable approximation) that Mirror Array 2 must subtend an angle $2 \theta$ at the lowest element in Mirror Array 1, so that Paths 1 and 2 may both be selected. This constraint implies a minimum separation between the mirror arrays, but leaves the tilt angle $\phi$ of each array undefined. There is clearly an advantage to reducing $\phi$ as shown in Fig. 4(b), so that the beams propagating between the mirrors just clear the lenses. If this is done, the angle of incidence on each mirror is reduced. The total path length between the lenses is then the sum of the three terms $L_{A}, L_{B}$, and $L_{C}$. If minimizing the path length is the only issue, and $L_{B}$ is roughly fixed as described above, there are advantages in choosing a large tilt angle as shown in Fig. 4(c), because this allows $L_{A}$ and $L_{C}$ to be reduced. Some practical layouts have used this approach [22].

There are, however, several advantages to a small tilt angle. Because the beams strike the mirrors at near-normal incidence, smaller mirrors are needed. Polarization-dependent effects are minimized, as are astigmatic effects caused by mirror curvature. The mirrors are all located at similar points along each path, and the path-length variation during switching is low. The eventual choice of tilt angle is therefore likely to represent a compromise.

Here, we are not concerned with the ideal choice of layout. Instead, we focus on a layout that allows major analytic simplifications, so that scaling laws may easily be derived. The chosen layout is shown in Fig. 4(d). Here, the mirror planes abut the lens arrays. For a small turn angle, it is simple to show that the tilt angle must also be small, and that the lengths $L_{A}, L_{B}$, and $L_{C}$ must all be approximately equal to $L$. Although this layout may not represent the optimum, it allows useful general conclusions to be derived easily. 
The mirrors are located on a regular pitch $P$, so that the overall width of the mirror array is $W=N P$. Assuming that small-angle approximations can be used, the width $W$ of the mirror array may be related to the distance $L$ and the angle $\theta$ by

$$
W \approx 2 L \theta .
$$

The total optical path $\zeta$ between the lenses is then

$$
\zeta \approx 3 L \approx 3 N P / 2 \theta \text {. }
$$

The number of mirrors $N$ across the array can then be related to $\theta, \zeta$, and $P$ by

$$
N \approx 2 \theta \zeta / 3 P .
$$

Equation (3) implies that the number of ports $N^{2}$ may be maximized for a given turn angle $\theta$ by minimizing the ratio $P / \zeta$. The beams should therefore have the smallest diameter that can be maintained over the distance between the lenses. Suitable beam parameters may be found by considering the propagation of Gaussian beams, solutions to the cylindrical wave equation that are realistic models for many bounded beams.

The electric field of a Gaussian optical beam is a function of its axial coordinate $z$ and its radial coordinate $r$, given by [39]

$$
E(r, z)=A_{0} \exp \left\{-r^{2} / w^{2}\right\} \exp \left\{-j k_{0} r^{2} / 2 R\right\} .
$$

Here, the term $\exp \left\{-r^{2} / w^{2}\right\}$ describes the radial amplitude variation, while $\exp \left\{-j k_{0} r^{2} / 2 R\right\}$ is the radial variation in phase. The parameter $w$ is a radius at which the amplitude falls to $1 / e$ of its maximum. The parameter $R$ is a characteristic radius that describes the curvature of the phase front. $k_{0}$ is the propagation constant, given by

$$
k_{0}=2 \pi / \lambda
$$

where $\lambda$ is the optical wavelength.

The values of $w$ and $R$ vary with distance according to

$$
\begin{gathered}
w^{2}=w_{0}^{2}\left\{1+\left(z / z_{0}\right)^{2}\right\} \\
R=z\left\{1+\left(z_{0} / z\right)^{2}\right\} .
\end{gathered}
$$

The parameter $w_{0}$ is the minimum beam radius, which is located at $z=0$. Similarly, $z_{0}$ is a characteristic distance measured in the direction of propagation, given by

$$
z_{0}=k_{0} w_{0}^{2} / 2
$$

Near the waist $\left(z \ll z_{0}\right)$, the beam is narrow and the phase front is flat. Far from the waist $\left(z \gg z_{0}\right)$, the beam diverges as a bounded spherical wave.

To find the parameters of the Gaussian beam that has the smallest final width for a particular propagation distance $z$, we write the variation in beam width as

$$
w^{2}=w_{0}^{2}+4 z^{2} /\left(k_{0}^{2} w_{0}^{2}\right) .
$$

Differentiating (8), we obtain

$$
2 w d w /\left.d w_{0}\right|_{z=\mathrm{const}}=2 w_{0}-8 z^{2} /\left(k_{0}^{2} w_{0}^{3}\right) .
$$

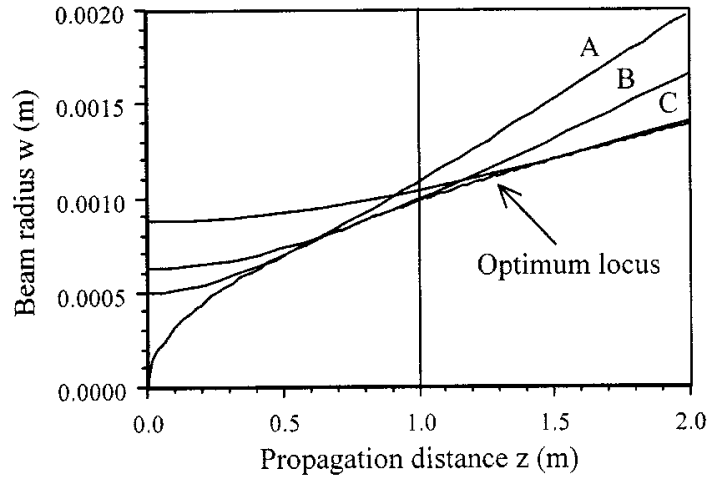

Fig. 5. Dotted lines: variation of $w$ with $z$ for three different Gaussian beams, assuming $\lambda=1.5 \mu \mathrm{m}$. Full line: limiting envelope obtained from (10) and (11).

At the minimum, the right-hand side of (9) must be zero, so that

$$
w_{0}=\sqrt{ }\left\{2 z / k_{0}\right\}=\sqrt{ }\{\lambda z / \pi\} .
$$

Equation (10) shows that the optimum waist radius $w_{0}$ scales as the square root of the required propagation distance $z$. For this value of $w_{0}$, the final beam radius is

$$
w=w_{0} \sqrt{ } 2 .
$$

The final value of the beam radius is therefore always related to the initial value by a simple constant, independent of the distance $z$. Comparing (6) and (11), we see that $z$ exactly equals the characteristic distance $z_{0}$. Near-field and far-field approximations are therefore both inappropriate, because the beam propagates to an intermediate distance.

To illustrate these points graphically, Fig. 5 compares the variation of $w$ with $z$ for three different Gaussians, to try to determine the optimum waist size for a fixed propagation distance of, say, $1 \mathrm{~m}$. A wavelength of $1.5 \mu \mathrm{m}$ is assumed. Beam A (with a waist radius of $w_{0}=0.5 \mathrm{~mm}$ ) is narrowest to begin with, but expands too rapidly. Beam C (with $w_{0}=0.875 \mathrm{~mm}$ ) is too wide to begin with, although it spreads more slowly. Beam B (with $w_{0}=0.625 \mathrm{~mm}$ ) has an intermediate waist and divergence, and is closest to the optimum at $1 \mathrm{~m}$.

The variation of $w$ with $z$ predicted by (10) and (11) is also shown. This envelope makes a tangent contact to curve $B$ at a point close to $z=1 \mathrm{~m}$. In fact, because it touches the extremes of all possible Gaussian beam variations, it must define the narrowest Gaussian that can be propagated over a fixed distance. The existence of an optimized beam does not appear to have been highlighted before, and it provides a convenient framework for deriving analytic limits to cross-connect performance.

The beam radius may be held within the final value $w$ over twice the distance $z$ by prefocusing the beam so that it first converges to a waist and then diverges, as shown in Fig. 6(a) (see, e.g., [18]). This beam is then the narrowest that may propagate between the two lenses, as shown in Fig. 6(b). In this case, the distance $z$ is related to the path length by

$$
\zeta=2 z_{0} .
$$

Fig. 6(b) also shows an input lens used to convert the beam emerging from an input fiber into the desired Gaussian, and an output lens to focus this beam into an output fiber. The focal 


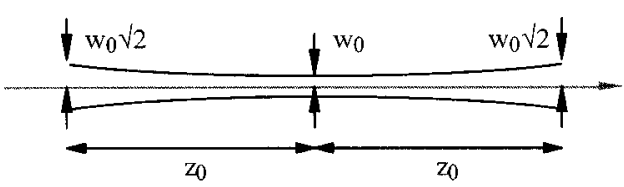

a)

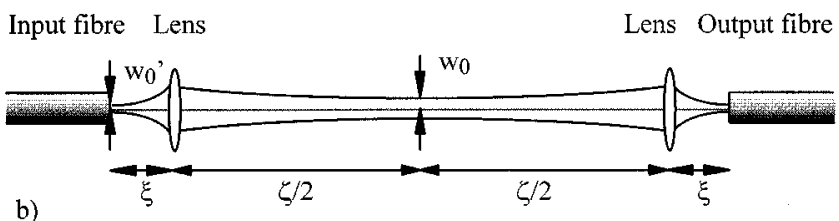

b)
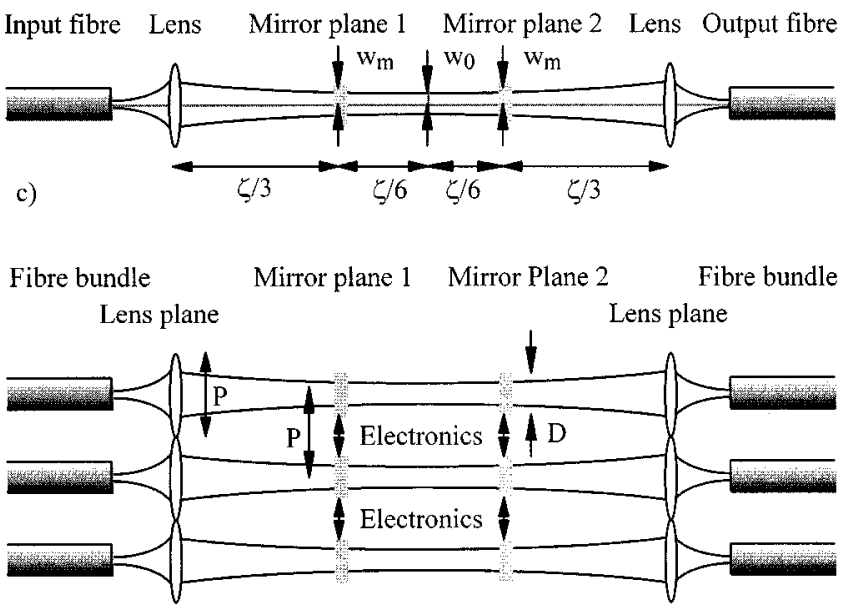

d)

Fig. 6. (a) Symmetric optimized Gaussian. (b) Creation of an optimized Gaussian from a fiber. (c) Unfolded model of a single connection in a cross connect. (d) Multiple connections.

length and position of the lenses must be specified correctly for high efficiency.

Single-mode fiber has a characteristic propagation mode that can be approximated well by a Gaussian with a mode field diameter of $\approx 8 \mu \mathrm{m}$ at a wavelength of $1.5 \mu \mathrm{m}$ [40]. Thus, the fiber emits a Gaussian with a waist radius $w_{0}^{\prime} \approx 4 \mu \mathrm{m}$. The lens must therefore transform one Gaussian beam with a waist radius $w_{0}^{\prime}$ a distance $\xi$ in front of the lens into another with a waist radius $w_{0}$ a distance $\zeta / 2$ behind it, as shown in Fig. 6(b). Unfortunately, the use of simple imaging formulas based on the sizes of the object and image will prove inaccurate, because neither lies in the far field of the lens. However, accurate analytic relations for the focal length $f$ of the required lens are known from earlier studies of Gaussian beam transformation [39]. The relevant equations are

$$
\begin{aligned}
\zeta / 2 & =f \pm M \sqrt{ }\left\{f^{2}-f_{0}^{2}\right\} \\
\xi & =f \pm 1 / M \sqrt{ }\left\{f^{2}-f_{0}^{2}\right\}
\end{aligned}
$$

Here, the parameters $M$ and $f_{0}$ are given by

$$
\begin{aligned}
& M=w_{0} / w_{0}^{\prime} \\
& f_{0}=(\pi / \lambda) w_{0} w_{0}^{\prime} .
\end{aligned}
$$

From (13), the focal length $f$ is the solution of the quadratic

$$
\left\{M^{2}-1\right\} f^{2}+\zeta f-\left\{M^{2} f_{0}^{2}+\zeta^{2} / 4\right\}=0 .
$$

Once $f$ is known, the distance $\xi$ may then be found from (13).

In fact, for a large magnification (which is normally the case), a simple solution may easily be obtained. Assuming that $M \gg 1$, (15) approximates to

$$
M^{2} f^{2}-\left\{M^{2} f_{0}^{2}+\zeta^{2} / 4\right\} \approx 0 .
$$

Combining (10), (12), and (14), we can also obtain

$$
M f_{0}=(\pi / \lambda) w_{0}^{2} \approx \zeta / 2 .
$$

Combining (16) and (17) and approximating (13) yields the final result.

$$
\begin{aligned}
& f \approx \zeta /(M \sqrt{ } 2) \\
& \xi \approx f .
\end{aligned}
$$

The coupling lenses are therefore not arbitrary, but must be chosen to match the rest of the system. The focal length is simply related to the path length and the system magnification. The fiber output facet must be placed close to the focal point of the input lens, and then moved axially by a small distance, so that an intermediate focus is created between the two lenses. By symmetry, the lens required for output coupling must have the same focal length, and the output fiber must be placed at the same distance from it.

Fig. 6(c) shows an unfolded model of a single connection in the switch. The mirrors lie at regular intervals between the lenses, so that the distance $z_{m}$ from the waist to each mirror is

$$
z_{m}=\zeta / 6=z_{0} / 3
$$

From (6), the beam radius $w_{m}$ at the mirror must therefore be given by

$$
w_{m}=w_{0} \sqrt{ }\{10 / 9\}=1.054 w_{0} .
$$

Equation (20) implies that the beam radius at each mirror is smaller than the maximum value $w=w_{0} \sqrt{ } 2$ at the lenses. If an entire switch is constructed from an array of similar optical connections, as shown in Fig. 6(d), there will therefore be sufficient space between the mirrors for local electronics. The pitch $P$ is governed by the requirement that the beams pass through each channel without significant attenuation. We now consider this aspect.

Equation (4) shows that the amplitude of a Gaussian beam is maximum at $r=0$, and falls gradually to zero as $r$ increases. When the beam is passed through an aperture of finite radius $\rho$, as provided by a lens or a mirror, some power must be lost. The throughput efficiency $\eta(\rho)$ can be estimated by integration, as

$$
\begin{aligned}
\eta(\rho) & \left.=\int_{0}^{\rho}|E(r)|^{2} 2 \pi r d r\right] \div\left\{\int_{0}^{i n f}|E(r)|^{2} 2 \pi r d r\right\} \\
& =1-\exp \left(-2 \rho^{2} / w^{2}\right) .
\end{aligned}
$$

For $\rho=w$, we obtain $\eta=0.865$, corresponding to a loss of $0.63 \mathrm{~dB}$. For $\rho=w \sqrt{ } 2$, the loss falls to $0.08 \mathrm{~dB}$. The attenuation may be reduced to a suitable value by taking the radii of any 
TABLE I

DESIGN Formulas and SCALING LaWS FOR MirRor Rotation Cross CONNECT SWITCHES

\begin{tabular}{llll}
\hline Parameter & Symbol & Formula & Scales as \\
\hline Characteristic distance & $\mathrm{z}_{0}$ & $9 \alpha^{2} \mathrm{~N}^{2} \lambda / 2 \pi \theta^{2}$ & $\mathrm{~N}^{2}, \alpha^{2}$ \\
Total path length & $\zeta$ & $2 \mathrm{z}_{0}$ & $\mathrm{~N}^{2}, \alpha^{2}$ \\
Beam radius at waist & $\mathrm{w}_{0}$ & $\sqrt{ }\left\{\lambda \mathrm{z}_{0} / \pi\right\}$ & $\mathrm{N}^{1}, \alpha^{1}$ \\
Beam radius on mirror & $\mathrm{w}_{\mathrm{m}}$ & $\sqrt{ }\{10 / 9\} \mathrm{w}_{0}$ & $\mathrm{~N}^{1}, \alpha^{1}$ \\
Mirror diameter & $\mathrm{D}$ & $2 \alpha \mathrm{w}_{\mathrm{m}}$ & $\mathrm{N}^{1}, \alpha^{2}$ \\
Mirror area fill factor & $\mathrm{F}$ & $5 \pi / 36$ & $\mathrm{~N}^{0}, \alpha^{0}$ \\
Beam radius at lens & $\mathrm{w}$ & $\mathrm{w}_{0} \sqrt{2}$ & $\mathrm{~N}^{1}, \alpha^{1}$ \\
Lens diameter & $\mathrm{P}$ & $2 \alpha \mathrm{w}$ & $\mathrm{N}^{1}, \alpha^{2}$ \\
Lens magnification & $\mathrm{M}$ & $\mathrm{w}_{0} / \mathrm{w}_{0}$, & $\mathrm{N}^{1}, \alpha^{1}$ \\
Lens focal length & $\mathrm{f}$ & $\zeta /(\mathrm{M} \sqrt{2})$ & $\mathrm{N}^{1}, \alpha^{1}$ \\
Lens numerical aperture & $\mathrm{NA}$ & $\mathrm{P} / 2 \mathrm{f}$ & $\mathrm{N}^{0}, \alpha^{1}$ \\
\hline
\end{tabular}

apertures to be a suitably large multiple $\alpha$ of the beam radius $w$. The lens pitch $P$ and mirror diameter $D$ should therefore be chosen so that

$$
\begin{aligned}
& P=2 \alpha w \\
& D=2 \alpha w_{m} .
\end{aligned}
$$

Using (22), we can define a linear mirror "fill factor" as the ratio of the mirror diameter to the pitch (i.e., as $F_{L}=D / P$ ). In a similar way, we define an area fill factor as the ratio of the mirror area to the pitch squared (i.e., as $F_{A}=\pi D^{2} / 4 \div P^{2}$ ). This gives

$$
\begin{aligned}
& F_{L}=\sqrt{ }(5 / 9)=0.745 \\
& F_{A}=5 \pi / 36=0.436 .
\end{aligned}
$$

Equation (23) shows that a reasonable fraction $\left(1-F_{A}=0.564\right)$ of the mirror plane area will indeed be available for local sense and drive electronics.

Combining (3) with (10)-(12) and (22), a closed-form expression for the number of mirrors $N$ across the array may be obtained as

$$
N=(\theta / 3 \alpha) \sqrt{ }(\pi \zeta / \lambda)
$$

For a given wavelength $\lambda$, mirror turn angle $\theta$, and optical path length $\zeta$, and assuming a particular value for the parameter $\alpha$, (24) represents the upper limit to the port count that may be achieved in this type of $N \times N$ (or 3-D $1 \times N$ ) mirror-rotation cross connect.

Alternatively, (24) may be inverted to find the path length needed for a given wavelength and turn angle, again assuming a particular value for $\alpha$, as

$$
\zeta=9 \alpha^{2} N^{2} \lambda / \pi \theta^{2} \text {. }
$$

Equation (25) shows that the path length must increase with the square of the number of ports $N$ in an $N \times N$ cross connect.
Because the layouts of $N \times N$ and $N^{2} \times N^{2}$ mirror-rotation switches are similar, the design formulas above are relevant to both types. Squaring (24) yields

$$
N^{2}=\left(\pi / 9 \alpha^{2} \lambda\right) \zeta \theta^{2}
$$

Equation (26) is a similar limit to the port count in this type of $N^{2} \times N^{2}$ (or 3-D $2 N$ ) switch.

The path length $\zeta$ required in an $N^{2} \times N^{2}$ cross connect may again be obtained from (25). In this case, $\zeta$ must increase linearly with the number of ports $N^{2}$. This result shows that an $N^{2} \times N^{2}$ mirror-rotation switch is generally more effective than a similar $N \times N$ switch, because the path length required for a similar number of ports is always smaller. The difference in path length may be considerable for large $N$.

In Table I, we combine all the results above in the form of a set of scaling laws, which show how the main parameters of this particular type of $N^{2} \times N^{2}$ cross connect depend on $N$ and $\alpha$. In Table II, we calculate the main parameters, assuming that the mirror turn angle is limited to $0.1 \mathrm{rad}$ or $6^{\circ}$. The switch is designed to operate at a $1.5-\mu \mathrm{m}$ wavelength, using fibers whose mode field radius is $4 \mu \mathrm{m}$. The number of ports is $N^{2}=1024$, so that $N=32$. The results are given for two different values of $\alpha$. The system with the larger value of $\alpha$ will have lower loss due to the effect of any apertures, but a longer optical path.

We now return briefly to the point made at the beginning of this section, namely, the existence of alternative layout possibilities. If, for example, the layout is as shown in Fig. 4(b) or (c), the three sections of the optical path are no longer equal. A similar analysis may be carried still be carried out, however. For example, when $\phi$ is no longer equal to $\theta$, but may still be considered a small angle, we may write $\phi=\beta \theta$ where $\beta$ is a constant. In this case, the scaling law for the optical path (25) modifies to

$$
\zeta=\gamma \alpha^{2} N^{2} \lambda / \pi \theta^{2}
$$


TABLE II

Design Parameters of AN $N^{2} \times N^{2}$ Cross Connect, With $\theta=0.1 \mathrm{rad}, \lambda=1.5 \mu \mathrm{m}$, $w_{0}^{\prime}=4 \mu \mathrm{m}$, AND $N^{2}=1024$, FOR Two DifFERENT VALUES OF $\alpha$

\begin{tabular}{llll}
\hline Parameter & Symbol & Value, $\alpha=1$ & Value, $\alpha=\sqrt{2}$ \\
\hline Total path length & $\zeta$ & $0.44 \mathrm{~m}$ & $0.88 \mathrm{~m}$ \\
Beam radius at waist & $\mathrm{w}_{0}$ & $0.324 \mathrm{~mm}$ & $0.458 \mathrm{~mm}$ \\
Beam radius on mirror & $\mathrm{w}_{\mathrm{m}}$ & $0.342 \mathrm{~mm}$ & $0.483 \mathrm{~mm}$ \\
Mirror diameter & $\mathrm{D}$ & $0.684 \mathrm{~mm}$ & $1.366 \mathrm{~mm}$ \\
Beam radius at lens & $\mathrm{W}$ & $0.458 \mathrm{~mm}$ & $0.648 \mathrm{~mm}$ \\
Lens diameter & $\mathrm{P}$ & $0.916 \mathrm{~mm}$ & $1.833 \mathrm{~mm}$ \\
Lens magnification & $\mathrm{M}$ & 81.03 & 114.59 \\
Lens focal length & $\mathrm{f}$ & $3.81 \mathrm{~mm}$ & $5.39 \mathrm{~mm}$ \\
Lens numerical aperture & $\mathrm{NA}$ & 0.119 & 0.169 \\
Fibre-lens separation & $\xi$ & $3.84 \mathrm{~mm}$ & $5.43 \mathrm{~mm}$ \\
\hline
\end{tabular}

Here, the constant multiplier is $\gamma=(1+2 / \beta)^{2}$. Equation (27) clearly has the same general form as (25), suggesting that the main conclusions drawn earlier are still valid. For $\beta=1$, we obtain $\gamma=9$ as before. However, if $\beta=2$, we obtain $\gamma=4$, suggesting that a useful reduction in optical path can be obtained from this more efficient arrangement.

\section{NUMERICAL ALGORITHM}

The propagation of a Gaussian beam is conveniently modeled in terms of a complex beam parameter $q=z+j z_{0}$ [39], which allows the field in (4) to be written as

$$
E(r, z)=A_{0} \exp \left\{-j k_{0} r^{2} / 2 q\right\} .
$$

Here, the beam width $w$ and the radius of curvature $R$ of the phase front are related to $q$ by

$$
\begin{aligned}
1 / w^{2} & =-\left(k_{0} / 2\right) \operatorname{Im}(1 / q) \\
1 / R & =\operatorname{Re}(1 / q) .
\end{aligned}
$$

The effect of propagation through a distance $z$, or through a lens of focal length $f$, is to modify $q$. The new value $q$ is given by the $A B C D$ rule [41], [42].

$$
q^{\prime}=\{A q+B) /\{C q+D\} .
$$

Here, the coefficients $A, B, C$, and $D$ take the following values:

$$
\begin{array}{lcccc} 
& A & B & C & D \\
\text { Propagation through a distance } z & 1 & z & 0 & 1 \\
\text { Propagation through a lens } & 1 & 0 & -1 / f & 1 .
\end{array}
$$

Equations (29)-(31) may be used to simulate propagation through an unfolded cross connect. If any mirror surface is spherically curved, its effect may also be modeled by replacing it with an equivalent spherical lens, and using the $A B C D$ algorithm once more. A lens of focal length $f=r / 2$ may simulate a concave mirror of radius $r$, and so on.

The throughput efficiency will be affected by any changes in the beam parameters that alter the spot focused onto the output fiber, such as apodization and defocus. The efficiency $\eta$ is found from the overlap between the output field $E_{\text {out }}$ and the fiber mode $E_{\text {fiber }}$ [43].

$$
\eta=\left|\left\langle E_{\text {out }}, E_{\text {fiber }}\right\rangle\right|^{2} /\left\{\left\langle E_{\text {out }}, E_{\text {out }}\right\rangle\left\langle E_{\text {fiber }}, E_{\text {fiber }}\right\rangle\right\} .
$$

Here, each term $\left\langle E_{1}, E_{2}\right\rangle$ is an overlap integral of the form

$$
\left\langle E_{1}, E_{2}\right\rangle=\iint E_{1} E_{2} * d a .
$$

The symbol $*$ denotes complex conjugate, and the double integral is taken over the whole of the transverse cross section.

In the examples that follow, we assume that any apertures may be neglected (although their effect is simple to insert), and concentrate on the effect of defocus in the output spot. In this case, the output field and fiber mode are both Gaussian beams with a common axis, and we may write

$$
\begin{aligned}
E_{\text {out }} & =A_{1} \exp \left\{-r^{2} / w_{\text {out }}^{2}\right\} \exp \left\{-j k_{0} r^{2} / 2 R_{\text {out }}\right\} \\
E_{\text {fiber }} & =A_{2} \exp \left\{-r^{2} / w_{\text {fiber }}^{2}\right\}
\end{aligned}
$$

to get

$$
\begin{aligned}
\eta=4 w_{\text {out }}^{2} w_{\text {fiber }}^{2} /\left\{\left\{w_{\text {out }}^{2}\right.\right. & \left.+w_{\text {fiber }}^{2}\right\}^{2} \\
& \left.+\left\{k_{0} w_{\text {out }}^{2} w_{\text {fiber }}^{2} / 2 R_{\text {out }}\right\}^{2}\right\} .
\end{aligned}
$$

When $w_{\text {out }}=w_{\text {fiber }}$ and $R_{\text {out }}$ is infinite (i.e., when the size of the focal spot at the output fiber exactly matches the fiber mode size and the output phase front is plane), we obtain $\eta=1$. Conversely, if $w_{\text {out }} \neq w_{\text {fiber }}$ or $R_{\text {out }} \neq 0$, the throughput efficiency is reduced. 
Input fibre Lens Mirror plane 1 Mirror plane 2 Lens Output fibre

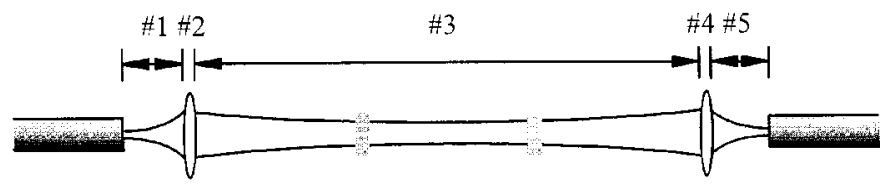

a)

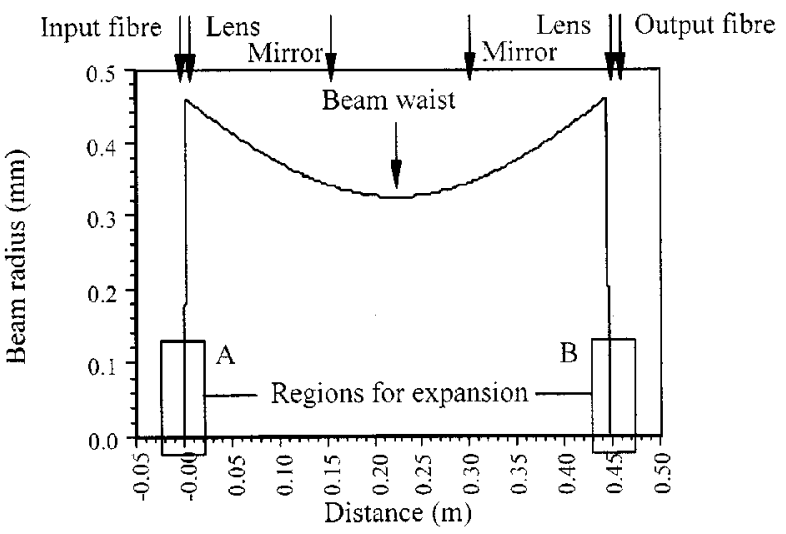

b)

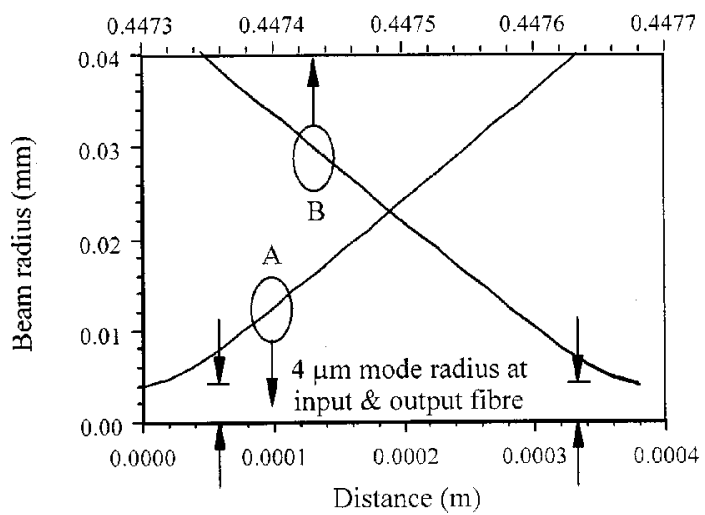

Fig. 7. (a) Model of a single channel of a cross connect. (b) Variation of beam radius with position. (c) Expanded variations near the input and output fiber.

\section{NUMERICAL EXAMPLES}

We now use the algorithms of Section III to illustrate propagation through the cross connect of Fig. 4(d). We assume that the layout is based on the optimized Gaussian beam as described in Section II. We assume the parameters of Table II, for the particular case when $\alpha=1$ (so that the separation between the lenses is $0.44 \mathrm{~m}$ ).

\section{A. Ideal Optical Arrangement}

We begin with an ideal case, when the fibers are positioned correctly, the lens separation is correct, and the mirrors are flat. Fig. 7(a) shows a model of a single channel, which can be separated into five regions for the calculation. Fig. 7(b) shows the variation of the beam radius through the system. The beam expands rapidly as it emerges from the input fiber. When it reaches the first lens, it is focused to a waist at the midpoint It expands again as it propagates to the second lens, where it is focused onto the output fiber. Fig. 7(c) shows enlargements near the two fibers. The simulation confirms all the details in Section II; the beam varies as expected, and the beam size at the output matches the value at the input.

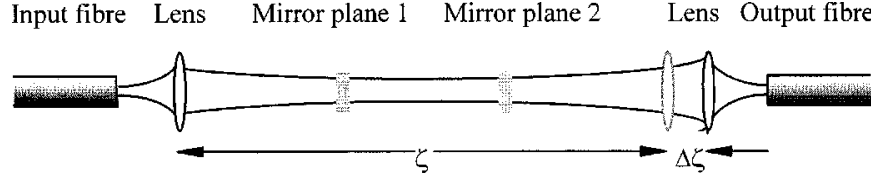

a)

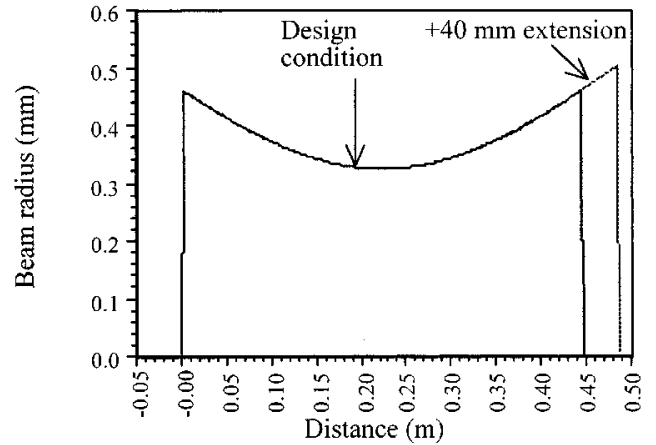

b)

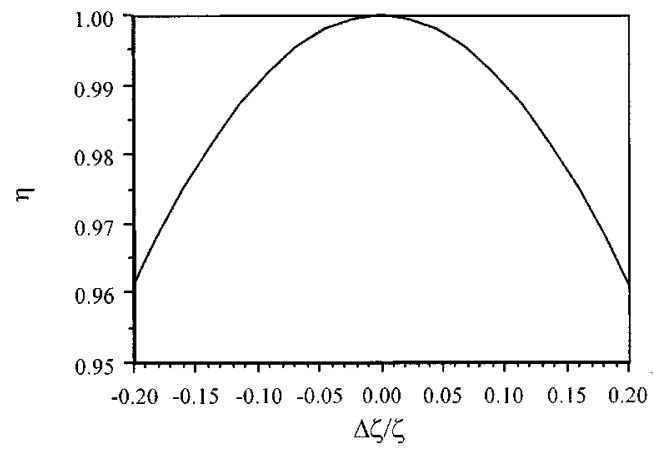

Fig. 8. (a) Model of axial extension in a cross connect. (b) Variation of beam radius with position. (c) Variation of throughput efficiency with axial extension.

\section{B. Effect of Optical Path Variations}

We now consider a nonideal case, when the separation between the lenses is altered from the design value (for example, through the selection of a different port-to-port connection by the mirrors). This may be modeled by an increase in the distance $\zeta$ by $\Delta \zeta$ as shown in Fig. 8(a). Fig. 8(b) shows the variation of beam radius with position for 1) $\Delta \zeta=0$, and 2) $\Delta \zeta=+40 \mathrm{~mm}$. The two variations are clearly similar; however, the beam radius at the second lens is slightly larger in 2) because of the increased optical path.

By numerical simulation, it can be shown that the effect of axial extension on throughput efficiency is scalable, so that the allowable extension $\Delta \zeta$ for a given pathlength is directly proportional to $\zeta$. Fig. 8(c) shows the variation of $\eta$ with $\Delta \zeta / \zeta$. The efficiency reduces very slowly, so that high efficiencies are obtained even when $\Delta \zeta / \zeta$ approaches the initial mirror separation. Consequently, the cross connect will have high port-to-port uniformity.

\section{Effect of Mirror Curvature}

Because the mirrors are flat in the examples above, they do not affect the propagation of the beam. However, the use of a multilayer construction may result in curvature of the mirrors due to intrinsic stress or thermal expansion. We therefore consider a second nonideal case, when the first mirror is spherically curved. This situation can be modeled by inserting an equivalent lens, dividing the calculation into seven separate regions as shown in Fig. 9(a). 
Input fibre Lens Mirror plane 1 Mirror plane 2 Lens Output fibre

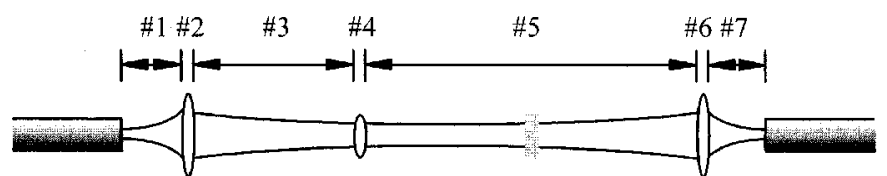

a)

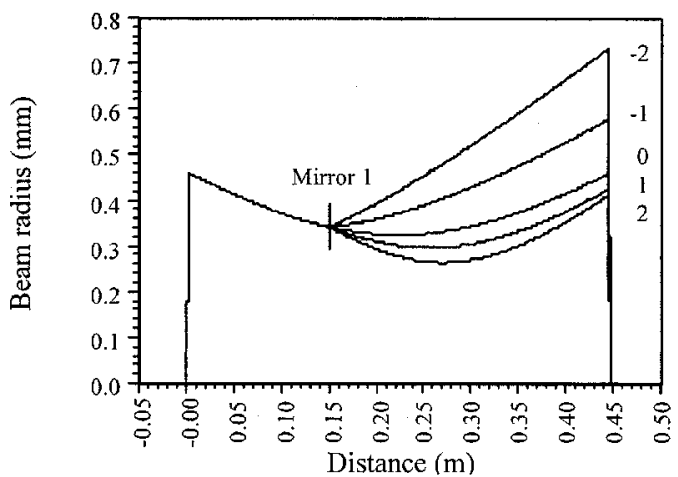

b)

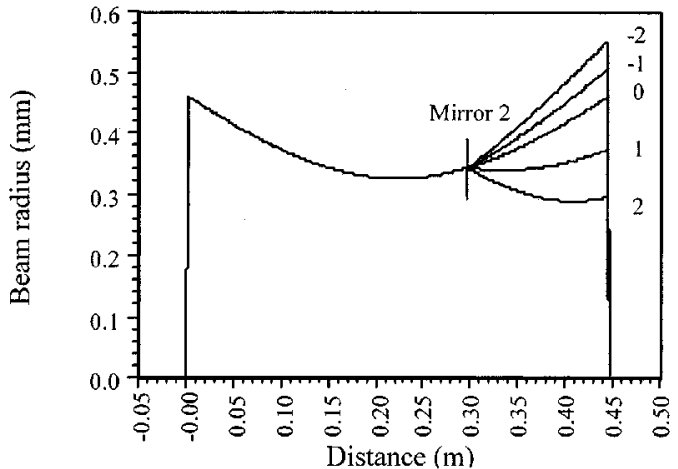

Fig. 9. (a) Model of cross connect with one curved mirror. (b) and (c) Variation of beam radius with position for different curvatures (in $\mathrm{m}^{-1}$ ) applied to mirrors 1 and 2 , respectively.

Fig. 9(b) shows the variation of beam radius with position, for different mirror curvatures $c=1 / 2 f$ in the range -2 to $+2 \mathrm{~m}^{-1}$. This corresponds to radii of curvature (ROC) in the range $|\mathrm{ROC}| \geq 50 \mathrm{~cm}$. The effect of convex curvature (negative $c$ and $f$ ) is to diverge the beam, making it too large to pass through the output lens without attenuation. The effect of concave curvature (positive $c$ ) is initially to decrease the divergence, partially focusing the beam. However, if the curvature is too large, the focusing effect is too great, and the beam diverges again before it reaches the output lens. After this, it will again be too large to pass without attenuation. Fig. 9(c) shows the corresponding result when only the second mirror is curved. Similar results are obtained, but the reduced optical path between the second mirror and the output lens allows the beam diameter to be held low for larger curvatures.

When both mirrors are curved, the system can be modeled using two equivalent lenses as in Fig. 10(a). Fig. 10(b) shows the variation of beam radius with position, for different curvatures applied equally to both mirrors. When both are convex, the beam diverges strongly. However, when both are concave, mirror 2 compensates for mirror 1 to some extent, and the beam is held within bounds for curvatures up to $\approx 4 \mathrm{~m}^{-1}$ (ROC $\geq 25 \mathrm{~cm}$ ).

Fig. 10(c) compares the throughput efficiency with mirror curvature, for the three cases before, namely, when curvature is
Input fibre Lens Mirror plane 1 Mirror plane 2 Lens Output fibre

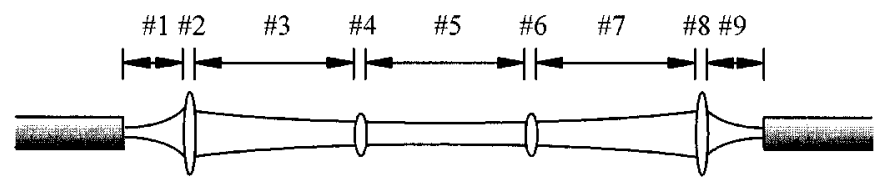

a)

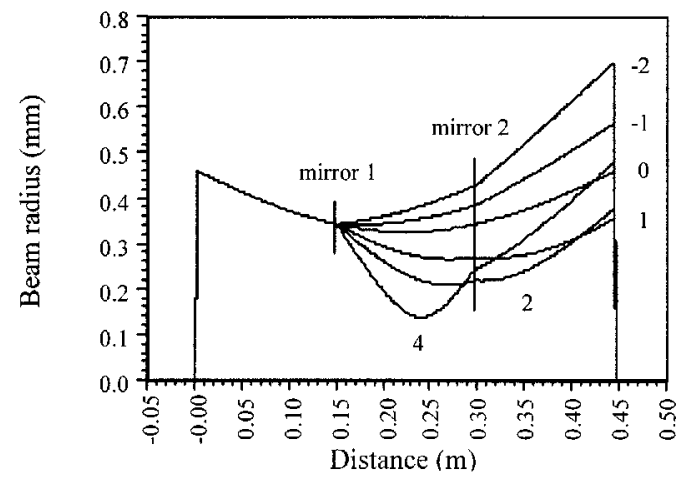

b)

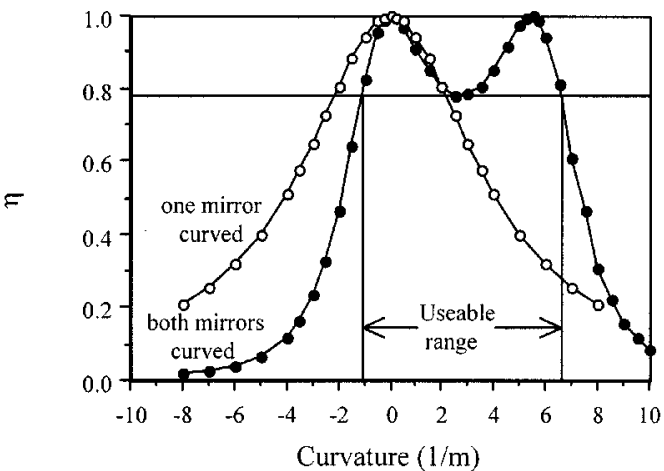

Fig. 10. (a) Model of cross connect with two curved mirrors. (b) Variation of beam radius with position for different curvatures (in $\mathrm{m}^{-1}$ ) applied to both mirrors. (c) Variation of efficiency with curvature, assuming that 1) one and 2) two mirrors are curved.

applied to 1) mirror 1 alone, 2) mirror 2 alone, and 3) both mirrors together. In all three cases, the reduction in efficiency is far greater than in Fig. 8(c), illustrating the importance of mirror flatness. Cases 1) and 2) give identical results, implying reciprocity. However, the increased divergence caused by curvature of mirror 1 suggests that the flattest mirrors available should be placed here.

For case 3), the reduction in efficiency caused by negative curvature of both mirrors is considerably larger than the corresponding result for either mirror alone. For increasingly positive mirror curvature, the throughput efficiency first falls slightly, then rises back to unity, and finally falls sharply once more. As a result, the efficiency is more constant over a wider range than in cases 1) and 2). In this example, the efficiency is more than $\eta \approx 0.8$ (corresponding to $1-\mathrm{dB}$ loss) up to a mirror curvature of $\approx 7 \mathrm{~m}^{-1}$ (ROC of $\left.\approx 14 \mathrm{~cm}\right)$.

This characteristic may be exploited to minimize the effect of mirror curvature, as follows. The curvature should be biased to the center of the usable range to minimize the effect of random changes (for example, due to variations in the intrinsic stress of coating layers). Alternatively, it should be biased to the right hand of the usable range to minimize the effect of systematic curvature (for example, due to heating). 
By further numerical simulation, it can be shown that the effect of mirror curvature on throughput efficiency is again scalable, and that the sensitivity to curvature is exactly proportional to the optical path length $\zeta$.

\section{Perfect Imaging With ImPerfect Components}

At first sight, it seems unlikely that a system constructed for perfect throughput with flat mirrors would also have $100 \%$ efficiency when both mirrors are curved. We now give an analytic proof of the perfect imaging result and calculate the required mirror curvature.

We first define a possible way for the imperfect system to operate. We begin by asserting that perfect throughput is obtained in the ideal case because the system is symmetric. The first lens transforms the beam leaving the input fiber into a Gaussian whose waist is located at the midpoint. The second lens reverses the transformation, presenting a beam at the output fiber that exactly mimics the fiber mode. For similar reasons, we guess that perfect throughput is obtained in a system with curved mirrors if it is also symmetric, with a midpoint waist. We then invoke the uniqueness of the transformation process, arguing that a Gaussian beam with the original parameters must still be presented at the second lens, because this lens must transform it into the original output field. If the original beam is to be presented at the second lens after transformation by a curved mirror, it must have emanated from a smaller midpoint waist than before, and been transformed by a concave mirror that restored its divergence to the original value.

Using these assumptions, Fig. 11(a) compares the original system with a similar perfect imaging system containing additional curved mirrors. For clarity, only the second half of each system is shown. The second curved mirror is modeled by an equivalent converging lens of focal length $f$. At the midpoint, we assume waist radii of $w_{0}$ in the original system and $w_{0}^{\dagger}$ (an unknown quantity) in the system with curved mirrors. The corresponding complex beam parameters are $q_{0}$ and $q_{0}^{\dagger}$.

For any Gaussian, the complex beam parameter must be purely imaginary at the waist, because the phase front is flat. We can therefore write $q_{0}=j k_{0} w_{0}^{2} / 2=j z_{0}$ (known) and $q_{0}^{\dagger}=j k_{0} w_{0}^{\dagger 2} / 2=j z_{0}^{\dagger}$ (unknown). After propagation to the mirror, the beam parameters modify as described by the $A B C D$ rules. For the original system, the new value is $q_{1}$; for the system with curved mirrors, it is $q_{1}^{\dagger}$ just before the mirror, and $q_{2}^{\dagger}$ just after, where

$$
\begin{aligned}
& q_{1}=q_{0}+z_{0} / 3 \\
& q_{1}^{\dagger}=q_{0}^{\dagger}+z_{0} / 3 \\
& q_{2}^{\dagger}=q_{1}^{\dagger} /\left\{-q_{1}^{\dagger} / f+1\right\} .
\end{aligned}
$$

If the beams leaving the mirror are to be the same in each system, we must have $q_{1}=q_{2}^{\dagger}$. Equating real and imaginary parts, we obtain

$$
\text { so that } \begin{aligned}
z_{0}^{\dagger} & =z_{0} / 9 \\
w_{0}^{\dagger} & =w_{0} / 3 \\
f & =(5 / 12) z_{0} .
\end{aligned}
$$

Equation (36) is a unique solution, which can define a single set of parameters for the modified system, whatever the path length.

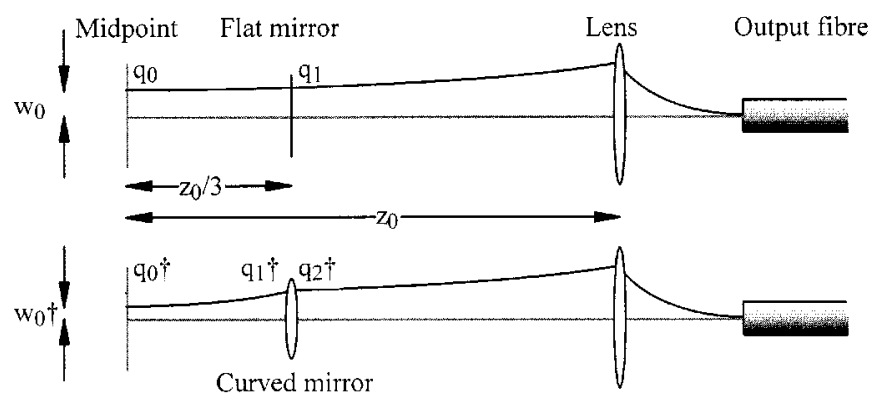

a)

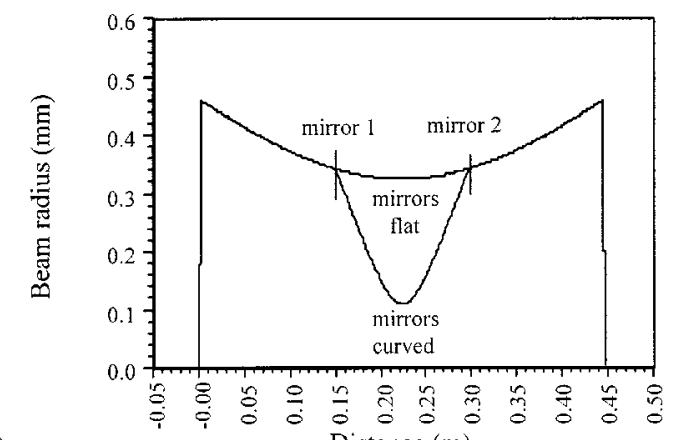

b)

Distance $(\mathrm{m})$

Fig. 11. (a) Model of propagation through the second half of a system with and without curved mirrors. (b) Variation of beam radius with position for the two perfect imaging cases.

The midpoint waist when the mirrors are curved is always one third the size obtained when they are flat. The focal length of the equivalent lens is always positive, so that the lens is converging, and $f$ is linearly proportional to $z_{0}$. The corresponding mirror radius and curvature are

$$
\begin{aligned}
& r=2 f=(5 / 6) z_{0} \\
& c=1 / r=(6 / 5) \times 1 / z_{0} .
\end{aligned}
$$

For $\zeta=0.44 \mathrm{~m}$ and $z_{0}=0.22 \mathrm{~m}$, we obtain $c=5.45 \mathrm{~m}^{-1}$, the value at which transmission through the distorted system is restored to unity in Fig. 10(c). Fig. 11(b) shows the variation of the beam radius through the distorted system, which is exactly as predicted.

\section{SUMMARY}

We have considered the design of compact mirror-rotation optical cross-connect switches. Using simple analysis, we have first shown that it is possible to define an optimum Gaussian beam to propagate through a particular distance. This beam has the smallest possible maximum diameter, and hence allows the closest packing of a bundle of beams. We have shown how the input and output optics should be chosen to create this beam from an input fiber mode and then couple it back into an output fiber.

Knowing the properties of the optimum Gaussian, we have calculated scaling laws for $N \times N$ and $N^{2} \times N^{2}$ cross connects (3-D $1 \times N$ and 3-D $2 N$ arrays) based on a particular architecture, which (although not necessarily optimal) allows useful simplifications. These laws show how the path length and other parameters are related to the throughput loss of an idealized system. Using a numerical simulation based on applying the $A B C D$ rules 
of Gaussian beam propagation to an unfolded model of the optical system, we have verified the analytic conclusions above. We have also considered a number of departures from the ideal, and shown how the additional losses caused by imperfections scale with optical path length. Particularly, we have shown that sensitivity to mirror curvature increases linearly with path length, so larger switches must have flatter mirrors. This result emphasizes the importance of the optimized Gaussian, which minimizes path length. Finally, we have shown a theoretical $100 \%$ transmission may be obtained for two values of mirror curvature, which allows a tolerant operating regime to be defined.

\section{ACKNOWLEDGMENT}

The author would like to thank J. Swift for his advice.

\section{REFERENCES}

[1] V. A. Aksyuk, D. J. Bishop, and P. L. Gammel, "Micro machined optical switch," U.S. Patent 5923 798, July 13, 1999.

[2] V. Aksyuk, B. Barber, C. R. Giles, R. Ruel, L. Stulz, and D. Bishop, "Low insertion loss packaged and fiber connectorised MEMS reflective optical switch," Electron. Lett., vol. 34, pp. 1413-1414, 1998.

[3] V. Aksyuk, B. Barber, C. R. Giles, and R. Ruel, "Low insertion loss packaged and fiber-connectorised Si surface-micromachined reflective optical switch," in Proc. Solid State Sensor and Actuator Workshop, Hilton Head, SC, June 1998, pp. 79-82.

[4] V. Dhuler, M. D. Walters, E. A. Hill, and A. B. Cowen, "Moveable microelectromechanical mirror structures," WIPO Patent 067 063, Nov. 9, 2000.

[5] V. A. Aksyuk, D. J. Bishop, J. E. Ford, and J. A. Walker, "Freespace optical bypass-exchange switch," U.S. Patent 5 943 454, Aug. 24, 1999.

[6] M. Mita, H. Fujita, and P. Helin, "Mechanical optical switch and method for manufacturing the same," WIPO Patent 0153 874, July 26, 2001.

[7] P. Helin, M. Mita, T. Bourouina, G. Reyne, and H. Fujita, "Self-aligned micromachining process for large-scale free-space optical cross connects," J. Lightwave Technol., vol. 18, pp. 1785-1791, Dec. 2000.

[8] H. Maekoba, P. Helin, G. Reyne, T. Bourouina, and H. Fujita, "Selfaligned vertical mirror and V-grooves applied to an optical-switch: Modeling and optimization of bi-stable operation by electromagnetic actuation," Sens. Actuators, vol. A87, pp. 172-178, 2001.

[9] C. Marxer, "Switching arrangement for a radiation guide," WIPO Patent 0157578 , Aug. 9, 2001.

[10] C. Marxer and N. F. de Rooij, "Micro-opto-mechanical $2 \times 2$ switch for single-mode fibers based on a plasma-etched silicon mirror and electrostatic actuation," J. Lightwave Technol., vol. 17, pp. 2-6, Jan. 1999.

[11] C. Marxer, Y. Girardin, and N. F. de Rooij, "4 $\times 4$ fiber optic matrix switch based on MOEMS," in Proc. 3rd Int. Conf. Micro Opto Electro Mechanical Systems, 1999, pp. 74-75.

[12] M. F. Dautartas, A. M. Benzoni, Y. C. Chen, G. E. Blonder, B. H. Johnson, C. R. Paola, E. Rice, and Y. H. Wong, "A silicon-based moving mirror optical switch," J. Lightwave Technol., vol. 8, pp. 1075-1085, Aug. 1992

[13] H. Toshiyoshi and H. Fujita, "Electrostatic micro torsion mirrors for an optical switch matrix," J. Microelectromech. Syst., vol. 5, pp. 231-237, Dec. 1996.

[14] L.-Y. Lin, "Fiber-optic free-space micromachined matrix switches," U.S. Patent 6215921 , Apr. 10, 2001.

[15] L. Y. Lin, E. L. Goldstein, and R. W. Tkach, "Angular precision enhancement in free-space micromachined optical switches," U.S. Patent 6292600 , Sept. 18, 2001

[16] L. Y. Lin, E. L. Goldstein, J. M. Simmona, and R. W. Tkach, "Free-space micromachined optical switches with submillisecond switching time for large-scale optical cross connects," IEEE Photon. Technol. Lett., vol. 10, pp. 525-527, Apr. 1998.

[17] L. Y. Lin and E. L. Goldstein, "Lightwave micromachines for optical cross connects," in Proc. 25th Eur. Conf. Optical Communication (ECOC), vol. I, Nice, France, Sept. 1999, pp. 114-117.

[18] L. Y. Lin, E. L. Goldstein, and R. W. Tkach, "Method and apparatus for low loss via geometrical optimization in free-space micro-machined optical switches," U.S. Patent 06317532 , Nov. 13, 2001

[19] A. Husain and L. Fan, "Micromachines optomechanical switching cell with parallel plate actuator and on-chip power monitoring," WIPO Patent 0073 840, Dec. 7, 2000.
[20] S. S. Lee, L. Y. Lin, and M. C. Wu, "Surface micromachined free-space fiber optic switches," Electron. Lett., vol. 31, pp. 1481-1482, 1995.

[21] S. S. Lee, L.-S. Huang, C.-J. Kim, and M. C. Wu, "Free-space fiberoptic switches based on MEMS vertical torsion mirrors," J. Lightwave Technol., vol. 17, pp. 7-13, Jan. 1999.

[22] P. M. Hagelin, U. Krishnamoorty, J. P. Heritage, and O. Solgaard, "Scalable optical cross-connect switch using micromachined mirrors," IEEE Photon. Technol.. Lett., vol. 12, pp. 882-884, July 2000.

[23] A. P. Neukermans and T. G. Slater, "Micromachined torsional scanner," U.S. Patent 5629790 , May 13, 1997.

[24] — - "Micromachined hinge having an integral torsion sensor," U.S. Patent 5648 618, July $15,1997$.

[25] A. P. Neukermans, T. G. Slater, and P. Downing, "Micromachined members coupled for relative rotation by torsion bars," U.S. Patent 6044705 , Apr. 4, 2000.

[26] V. A. Aksyuk and D. J. Bishop, "Self-assembling micro-mechanical device," U.S. Patent 5994 159, Nov. 30, 1999.

[27] V. A. Aksyuk, F. Pardo, C. A. Bolle, S. C. Arney, C. R. Giles, and D. J. Bishop, "Lucent Microstar micromirror array technology for large optical cross connects," Proc. SPIE, vol. 4178, pp. 320-324, 2000.

[28] A. Gasparyan, V. A. Aksyuk, P. A. Busch, and S. C. Arney, "Mechanical reliability of surface-micromachined self-assembling two-axis MEMS tilting mirrors," Proc. SPIE, vol. 4180, pp. 86-90, 2000.

[29] O. Degani, E. Socher, A. Lipson, T. Leitner, D. J. Setter, S. Kaldor, and Y. Nemirovsky, "Pull-in study of an electrostatic torsion microactuator," J. Microelectromech. Syst., vol. 7, pp. 373-379, Dec. 1998.

[30] J. D. Drake, J. H. Jerman, and J. D. Grade, "Micromachined mirror with stretchable restoring force member," U.S. Patent 6128 122, Oct. 3, 2000.

[31] J.-L. A. Yeh, J. Jiang, and N. C. Tien, "Integrated polysilicon and DRIE bulk silicon micromachining for an electrostatic torsional actuator," $J$. Microelectromech. Syst., vol. 8, pp. 456-465, Dec. 1999.

[32] B. Behrang and S. Pannu, "Optical switch employing biased rotatable comb drive devices and methods," WIPO Patent 0 173 935, Oct. 4, 2001.

[33] A. Scott, T. Davis, C. Lee, J. Chong, S. Miller, and K. Shaw, "Electrostatic actuator for microelectromechanical systems and methods of fabrication," WIPO Patent 0157902 , Aug. 9, 2001.

[34] A. Husain and L. Fan, "Micromachined optomechanical switching devices," WIPO Patent 0075710 , Dec. 14, 2000.

[35] M. J. Daneman, M.-H. Kiang, B. Behin, and K. Y. Lam, "Optical switch having equalized beam spreading in all connections," WIPO Patent 0165300 , Sept. 7, 2001.

[36] S. Gloeckner, A. Husain, and L. Fan, "Optomechanical matrix switches including collimator arrays," WIPO Patent 0073 842, Dec. 7, 2000.

[37] D. M. Burns and V. M. Bright, "Optical power induced damage to microelectromechanical mirrors," Sens. Actuators, vol. A70, pp. 6-14, 1998.

[38] O. B. Spahn, C. Tigges, R. Shul, S. Rodgers, and M. Polosky, "High optical power handling of pop-up microelectromechanical mirrors," in IEEE/LEOS Int. Conf. Optical MEMS, 2000, pp. 51-52.

[39] D. Marcuse, Light Transmission Optics. New York: Van Nostrand Reinhold, 1972.

[40] G. Agrawal, Fiber-Optic Communication Systems. New York: Wiley, 1992.

[41] H. Kogelnik, "Imaging of optical mode resonators with internal lenses," Bell. Syst. Tech. J., vol. 44, pp. 455-494, 1965.

[42] H. Kogelnik and T. Li, "Laser beams and resonators," Appl. Opt., vol. 5, pp. $1550-1567,1966$.

[43] R. R. A. Syms and J. R. Cozens, Optical Guided Waves and Devices. New York: McGraw-Hill, 1992.

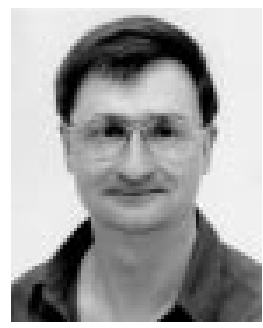

Richard R. A. Syms (M'98) was born in Norfolk, VA, in 1958. He received the B.A. degree in engineering science and the D.Phil. degree on volume holographic optical elements from Worcester College, Oxford, U.K., in 1979 and 1982, respectively.

He has been Head of the Optical and Semiconductor Devices Section in the Department of Electrical and Electronic Engineering, Imperial College London, U.K., since 1992 and Professor of Microsystems Technology since 1996. He has published approximately 80 papers and two books on holography, integrated optics, and microengineering. His current research interests are silica-on-silicon integrated optics and silicon-based MEMS. Recently, he has been developing microengineered mass spectrometers, frequency-tunable microactuators, and three-dimensional self-assembling microstructures. 